\title{
ASPIRATION OF A NONLINEAR ELASTIC SPHERICAL MEMBRANE
}

\author{
Rudenko O. V. ${ }^{*}$, Kolesnikov A. M. \\ ${ }^{\dagger}$ Southern Federal University, 344006, Rostov-on-Don, Bolshaya Sadovaya st, 105/42 \\ Alexey.M.Kolesnikov@gmail.com, zhbanova@sfedu.ru
}

Introduction. The cell membrane surrounds the heterogeneous material, which is called the cytoplasm. She is responsible for the regulation of exchange between the cell and its environment, for the maintenance of certain conditions of the internal environment, the protection of the cytoplasm, nucleus, and vacuole. The mechanical properties of the membrane (the deformation and stress in it) can play an important role in its functioning [1-3]. Based on experimental data it can be assumed that the membrane has a small resistance to bending. So the membrane material can be considered as highly elastic and incompressible [4]. Thus it is necessary use a nonlinear theory of membrane shells to construct its mathematical model.

In this investigation we simulate a spherical cell's aspiration into a micropipette. This procedure is used to study the cell membrane's mechanical properties, for example [1-3].

Main. The cell membrane is modeled by a thin membrane nonlinear elastic shell. The action of intracellular substances to the membrane is considered like a evenly distributed normal pressure. In the initial state, the shell has a constant thickness $h$ and radius $r_{0}$. The shell material is described by the potential energy in the form of Bartenev - Khazanovich for incompressible material

$$
W=2 \mu\left(\lambda_{1}+\lambda_{2}+\left(\lambda_{1} \lambda_{2}\right)^{-1}-3\right) .
$$

Here $\lambda_{1}$ and $\lambda_{2}$ are denote principal elongations multiplicity.

We assume that membrane's inner pressure is constant and the shell retains axial symmetry. The shell has a radius $r_{0}$ in the undeformed state (Fig. 1, a). The shell radius becomes $R_{0}$ under internal pressure (Fig. 1, b). The process of aspiration is to create a negative pressure in the tube, causing part of the shell is drawn into the tube radius $r_{p}$. At the initial stage, we assume that the shell is in contact with the tube only to coordinate line $s_{c}=$ const (Fig. 1, c). The contact area is increases with a significantly membrane's penetration into the tube. Interaction occurs with the inner wall of the tube (Fig. 1, d). Due to the fact that the shell has a convex shape, the penetration of the shell in the tube is already $L_{0}=R_{0}-\sqrt{\left(R_{0}\right)^{2}-\left(r_{p}\right)^{2}}$ at the initial time.

The process of aspiration can be controlled either by varying the pressure in the tube (Fig. 1, e) [1, 3], or volume change in the tube(Fig. 1, f) [5]. In the latter case it is assumed that this is due to the movement of the piston inside the tube [5].

At the first stage (weak aspiration (Fig. 1, c)), shell can be divided into two areas: $\mathrm{AC}-$ area outside the micropipette, $\mathrm{CB}$ - area outside the micropipette. Then, shell's contact with micropipette grows into a contact area $\mathrm{CD}$ (strong aspiration Fig.1.d), at a certain threshold pressure. In this case, the task is solved for three are- 
as: $\mathrm{AC}$ - area outside the micropipette, $\mathrm{BD}$ - area outside the micropipette, $\mathrm{CD}$ contact area.
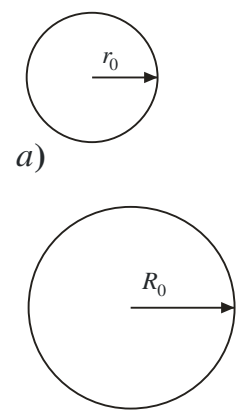

b)
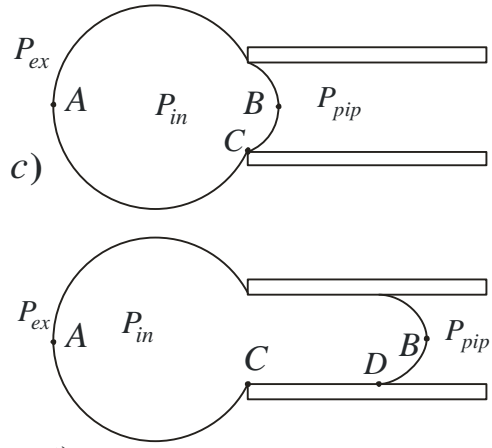

d)

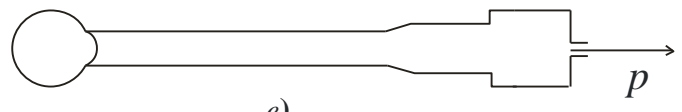

e)

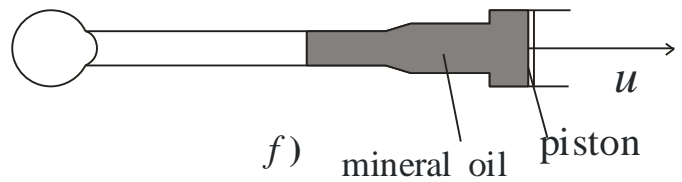

Figure 1 - Aspiration of the shell: a) - shell; b) - inflated shell; c) - a weak aspiration; d) - a strong aspiration e) - a process, which is controlled by varying the pressure; f) - a process controlled by the movement of the piston

Equilibrium equations are reduced to several systems of ordinary differential equations for the different areas, in the context of assumptions under consideration [6-8]. The boundary conditions are the conditions of continuity and smoothness of the solutions at the boundaries. Boundary value problem is solved numerically using Shooting method and Runge-Kutty methods.

Conclusion. The mechanical properties of the membrane and the cell as a whole can be inferred by the response shell on the deformation process [1]. Therefore, we are interested in the dependence of the depth of shell's suction $L$ and the parameters that create the aspiration conditions. Fig. 2 illustrates these dependences. Graph of $L$ and pressure in pipette $p_{\text {pip }}$ is illustrated by dashed line - for the case when the negative pressure creates in micropipette (Fig. 1, e). Solid line is illustrated the dependence $L$ and $U$, in the case when the shell is aspirated due to piston movement $U$ (Fig. 1, f).

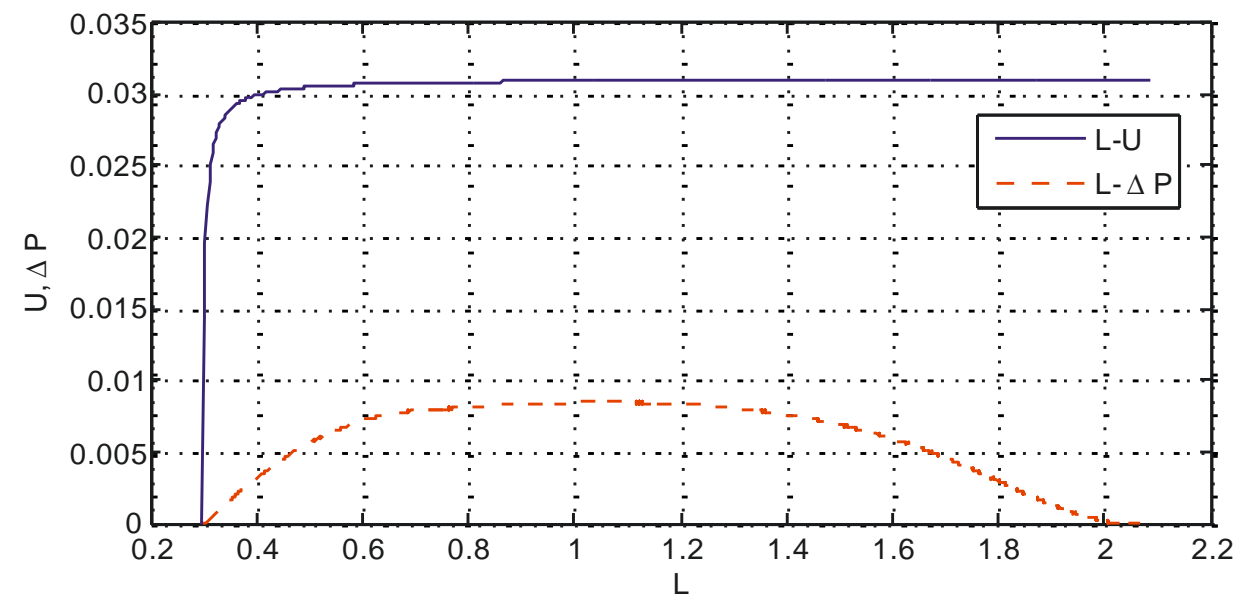

Figure 2 - Dashed line - dependence $L$ and $p_{\text {pip }}$; solid line - dependence $L$ and $U$. 
This research was supported by the Russian Foundation for Basic Research (grants 12-01-31431) and by theMinistry of Education and Science of the Russian Federation (contract 14.A18.21.0389).

\title{
References
}

1. Evans E., Yeung A. Apparent viscosity and cortical tension of blood granulocytes determined by micropipet aspiration // Biophys. J. - 1989. - Vol. 56. - P. 151 - 160.

2. Ruogang Zhao, Kristine Wyss, Craig A. Simmons Comparison of analytical andinverse finite element approach estoestimate cell viscoelastic properties by micropipette aspiration// J. Biomech. - 2009. - V. 42. - P. 2768 - 2773.

3. Eric M. Darling, Matthew Topel, Stefan Zausher, Thomas P. Vail, Farshid Guilak Viscoelastic properties of human mesenchymally-derived stem cells and primary osteoblast, chondrocytes, and adipocytes // J. Biomech. - 2008. - Vol. 41. - P. 454 - 464.

4. Sohail T. et al. Micropipette aspiration of an inflated fluid-filled spherical membrane. 2012.

5. Zhang X.-P. et al. Controlled Aspiration and Positioning of Biological Cells in a Micropipette. 2012.

6. Kolesnikov A. M., Zubov L. M. Large deformations of elastic momentless shells of revolution // Izvectija vuzov. Severo-Kavkazskij Region. Natural sciences. - 2004. - Vol. 1. - P. 33 - 37. (In Russian)

7. Kolesnikov A. M., Zubov L. M. Large bending deformations of a cylindrical membrane with internal pressure // ZAMM. - 2009. - Vol. 89, No. 4. - P. 288 - 305.

8. Zhbanova O. V. Model for the case of low aspiration nonlinear elastic spherical shell // Izvectija vuzov. Severo-Kavkazskij Region. Natural sciences. Special issue. - 2011. P. 28 - 30. (In Russian)

\section{NUMERAL MODELLING OF THE MECHANICAL CHARACTERISTICS OF THE COMPLEX «INTIMA - STENT»}

\author{
Zaretskiy A. P. \\ Bauman Moscow State Technical University, 105005, Moscow, $2^{\mathrm{d}}$ Baumanskaya, 5 \\ a.p.zaretskiy@gmail.com
}

\begin{abstract}
Stenting is one of the modern methods of surgical treatment of diseases of the coronary arteries. The essence of this procedure is an increasing of the vessel's interior diameter through the introduction of a thin-walled metal cylindrical shell - stent. Mechanical characteristics of the stent make the problem solvable. Restenosis is the principal problem of the stenting. Intima's (inner shell walls of a vessel) mechanical failure is one of the reasons of the restenosis. This surgical problem issues the challenge of the correct modeling of the mechanical characteristics of the coronary arteries and stent.

Let's consider the left coronary artery (LCA)and anterior descending coronary artery (ADCA) intima's and stent. Geometric features of the stent's wall in the context of the problem aren't important, that's why they were not taken into consideration. Thus, let's consider thin-walled cylindrical shell, which includes 3 sections with characteristics presented in the Table 1 ( $n-$ section's number).

The important step of the modeling is a creation of conditions:

1.An axisymmetric bending of a cylindrical shell is considered in this task.
\end{abstract}

